\title{
ETIC: Nord-Süd-Partnerschaft und dezentralisierte Zusammenarbeit
}

Pape Ndiaye Diouf

\section{CpenEdition}

\section{Journals}

Electronic version

URL: http://journals.openedition.org/sjep/557

DOI: $10.4000 /$ sjep.557

ISSN: 1663-9677

\section{Publisher}

Institut de hautes études internationales et du développement

\section{Printed version}

Date of publication: 1 novembre 2003

Number of pages: 153-155

ISSN: 1660-5926

\section{Electronic reference}

Pape Ndiaye Diouf, «ETIC: Nord-Süd-Partnerschaft und dezentralisierte Zusammenarbeit », Schweizerisches Jahrbuch für Entwicklungspolitik [Online], 22-2 | 2003, Online erschienen am: 10 Juni 2010, abgerufen am 08 September 2020. URL : http://journals.openedition.org/sjep/557 ; DOI : https://doi.org/10.4000/sjep.557 


\title{
ETIC: Nord-Süd-Partnerschaft und dezentralisierte Zusammenarbeit
}

\author{
Pape Ndiaye Diouf*
}

D as Projekt ETIC (Espaces Technologie Information Communication $^{1}$ ) baut auf einer kritischen und konstruktiven Analyse der Entwicklung im Bereich der Informations- und Kommunikationstechnologien (IKT) in verschiedenen westafrikanischen Ländern auf. Das Projekt stützt sich im Wesentlichen auf zwei Quellen: auf die Feldarbeiten, die zwischen 1998 und 2001 von einem Team des Centre National de la Recherche Scientifique (CNRS) von Bordeaux und des Institut Universitaire d'Etudes du Développement (IUED) in Senegal, Mali, Guinea, der Elfenbeinküste und Ghana durchgeführt wurden. Es stützt sich zweitens auf die wichtige Arbeit der gemeinschaftlichen und ländlichen Radiostationen, - hauptsächlich in Westafrika - welche in den letzten 20 Jahren Bauern, Fischer und andere Handwerker zu Wort kommen liessen und ihnen die Möglichkeit gaben, ihre Interessen besser zu vertreten und ihre Positionen zu stärken.

Die Untersuchungen leisteten einen zweifachen Beitrag:

$\square$ Eine Entmystifizierung: Obwohl die IKT in Afrika rasch an Terrain gewinnen, reicht ihre massive Ausbreitung nicht aus, um die Lebensbedingungen der grossen Mehrheit positiv und nachhaltig $\mathrm{zu}$ beeinflussen. In der Tat zeigt die Erfahrung, dass die Verfügbarkeit einer Technologie allein kein Garant für den gesellschaftlichen Fortschritt ist. Zudem besteht die Gefahr, dass die Eliten und eine privilegierte Bevölkerungsminderheit die IKT für sich allein beanspruchen. Dennoch sind zahlreiche Menschen und Institutionen in Afrika nach wie vor überzeugt - und sie handeln dementsprechend -, dass die IKT automatisch die Entwicklung ankurbeln und sich auf fast natürliche Weise in den gesellschaftlichen Modernisierungsprozess einfügen würden.

$\square$ Die Identifizierung gewisser Bedürfnisse und Forderungen, die von verschiedenen Akteuren (lokale Abgeordnete, Behördenvertreter, Unternehmer, Produzentenverbände sowie Jugend- und Frauenvereinigungen), welche sich zu Gunsten der Entwicklung ihrer Region oder ihres Landes engagieren, geäussert wurden.

Der Verband ETIC Nord-Sud, der 2001 auf Initiative von Afrikanern und Schweizern gegründet wurde, will durch einen innovativen und partnerschaftlichen Ansatz in Sachen IKT genau diesen Bedürfnissen

* Lehrbeauftragter am Institut universitaire d'études du développement (iuéd), Schweiz.

$1<$ www.etic-nordsud.org $>$. 
gerecht werden. Der Ansatz beruht auf dem Modell der dezentralisierten Entwicklungszusammenarbeit. Dieses Modell dürfte einen bedeutenden Beitrag zur nachhaltigen Entwicklung und zu einer spürbaren Verbesserung der Lebensbedingungen für die grosse Mehrheit leisten, und soll die afrikanischen Länder bei der Bewältigung sozialer, wirtschaftlicher, kultureller und politischer Herausforderungen unterstützen.

Im Unterschied zum allzu oft vorherrschenden Trend geht es darum, die menschliche und soziale Dimension der IKT in den Vordergrund zu rücken, und nicht die technischen Aspekte, die sowohl bei den Vorbereitungsarbeiten des Weltgipfels über die Informationsgesellschaft (WSIS) als auch im Rahmen von Nepad (New Partnership for Africa's Development) dominieren.

Aus dieser Sicht bestimmte ETIC zwei konkrete Prioritäten für seine Tätigkeit:

$\square$ Die Entwicklung der menschlichen Ressourcen und des Know-hows durch fachliche Beratung, Schulung und Bildung. Dadurch soll gewährleistet werden, dass die technischen Ressourcen den lokalen Gemeinschaften - namentlich in benachteiligten urbanen Gegenden und auf dem Land - zur Verfügung gestellt werden und ihnen einen Nutzen bringen. Ohne Bildungsanstrengungen und Massnahmen, welche die Gemeinschaften zum Umgang mit den IKT befähigen, ist ein erfolgreicher Einzug der IKT in Afrika nicht möglich;

$\square$ die Förderung des Zugangs zu hoch stehenden IKT-Ausrüstungen und -Dienstleistungen für die grosse Mehrheit der Bevölkerung.
Das Zielpublikum umfasst verschiedene Kategorien von Akteuren in den folgenden Sektoren: Primar- und Sekundarschulstufe, Jugend-, Frauenund Produzentenverbände, Vertreter von Kommunalbehörden und im Gesundheitsbereich tätige Personen.

Zur Verwirklichung seiner Ziele beabsichtigt der Verband ETIC, ein Netzwerk von so genannten „Etics“ zu errichten - dezentralisierte Räume, welche sowohl in Bezug auf den Zugang als auch auf die Bildungsmöglichkeiten mit den erforderlichen Ressourcen ausgestattet sind. Diese Räume sollen folgende verschiedenen Funktionen wahrnehmen bzw. ermöglichen:

$\square$ eine bessere Teilung von Macht und Ressourcen;

$\square$ eine verstärkte und besser koordinierte nationale und internationale Solidarität, indem direktere und (zeitlich wie auch räumlich),,verkürzte" Beziehungen zwischen den beteiligten Akteuren aus dem Süden und aus dem Norden geschaffen werden. Dies soll auf den Ebenen der Basis, der Verwaltung und der Entwicklungsbehörden geschehen;

$\square$ eine Intensivierung des Austauschs von Erfahrungen, Informationen und Ideen zwischen diesen Akteuren;

$\square$ eine Stärkung der Fähigkeiten der Gemeinschaften der Basis und der lokalen Behörden, zu verhandeln und die Bedürfnisse der Bevölkerung wahrzunehmen;

$\square$ eine grössere Transparenz bei der Verwaltung der Entwicklungsprojekte durch regelmässigere, systematischere und wirksamere $\mathrm{Be}$ gleitung, Kontrolle und Evaluation. 
Um die Umsetzung dieser Ziele zu gewährleisten, ist auch der Verband selbst gemäss den Grundsätzen der dezentralisierten Zusammenarbeit in Afrika und der Partnerschaft zwischen der Schweiz und Afrika organisiert. Bei der Feldarbeit stützt er sich auf nationale ETIC-Kommissionen - die senegalesische Kommission, welche die Pilotphase betreut, wurde 2002 ins Leben gerufen -, welche mit dem Vorstand des Verbandes in Genf in Kontakt stehen. Die einzelnen nationalen Komitees haben ihrerseits den Auftrag, lokale Ausschüsse mit Vertretern der sozialen Akteure der Orte oder Stadtviertel zu bilden, in denen der Verband tätig ist. 